\title{
A simpler energy transfer efficiency model to predict relative biological effect for protons and heavier ions
}

\author{
Bleddyn Jones * \\ Gray Laboratory, CRUK/MRC Oxford Insitute for Radiation Oncology, University of Oxford, Oxford, UK
}

\section{OPEN ACCESS}

Edited by:

Brian Timothy Collins, Georgetown University Hospital, USA

Reviewed by: Dalong Pang,

Georgetown University Hospital, USA Kevin Prise,

Queen's University Belfast, UK

${ }^{*}$ Correspondence: Bleddyn Jones, Gray Laboratory, CRUK/MRC Oxford Insitute for Radiation Oncology,

University of Oxford,

Oxford OX3 7DQ, UK bleddyn.jones@oncology.ox.ac.uk

Specialty section: This article was submitted to Radiation Oncology, a section of the

journal Frontiers in Oncology

Received: 13 May 2015 Accepted: 27 July 2015 Published: 11 August 2015

Citation:

Jones B (2015) A simpler energy transfer efficiency model to predict relative biological effect for protons and heavier ions.

Front. Oncol. 5:184. doi: 10.3389/fonc.2015.00184
The aim of this work is to predict relative biological effectiveness (RBE) for protons and clinically relevant heavier ions, by using a simplified semi-empirical process based on rational expectations and published experimental results using different ion species. The model input parameters are: $Z$ (effective nuclear charge) and radiosensitivity parameters $\alpha_{\llcorner}$and $\beta_{\mathrm{L}}$ of the control low linear energy transfer (LET) radiation. Sequential saturation processes are assumed for: (a) the position of the turnover point (LETU) for the LET-RBE relationship with $Z$, and (b) the ultimate value of $\alpha$ at this point $\left(\alpha_{\cup}\right)$ being non-linearly related to $\alpha_{L}$. Using the same procedure for $\beta$, on the logical assumption that the changes in $\beta$ with LET, although smaller than $\alpha$, are symmetrical with those of $\alpha$, since there is symmetry of the fall off of LET-RBE curves with increasing dose, which suggests that LETu must be identical for $\alpha$ and $\beta$. Then, using iso-effective linear quadratic model equations, the estimated RBE is scaled between $\alpha_{\cup}$ and $\alpha_{\llcorner}$and between $\beta_{\cup}$ and $\beta_{\llcorner}$from for any input value of $Z, \alpha_{L}, \beta_{L}$, and dose. The model described is fitted to the data of Barendsen (alpha particles), Weyrather et al. (carbon ions), and Todd for nine different ions (deuterons to Argon), which include variations in cell surviving fraction and dose. In principle, this new system can be used to complement the more complex methods to predict RBE with LET such as the local effect and MKM models which already have been incorporated into treatment planning systems in various countries. It would be useful to have a secondary check to such systems, especially to alert clinicians of potential risks by relatively easy estimation of relevant RBEs. In clinical practice, LET values smaller than LETU are mostly encountered, but the model extends to higher values beyond LET $U$ for other purposes such as radiation, protection, and astrobiology. Considerable further research is required, perhaps in a dedicated international laboratory, using a basket of different models to determine what the best system or combination of systems will be to make proton and ion beam radiotherapy as safe as possible and to produce the best possible clinical results.

Keywords: RBE, protons, ions, radiotherapy, radiobiology

\section{Introduction}

Positively charged particle therapy is increasing worldwide. Its numerous potential advantages in cancer therapy depend on the Bragg peak effect (1-4), but the increase in linear energy transfer (LET) causes enhanced biological effects which change normal tissue tolerances, as well as tumor control probabilities. LET, typically reported as kiloelectron volt per micrometer, refers to the ratio 
of energy released from a radiation beam per unit micrometer track length and is used as a measure of radiation quality. It can be expressed in two different ways, either as the mean or the dose averaged LET. Relative biological effect (RBE) is defined as the ratio of dose of a low LET radiation divided by the control high LET dose required for the same biological effect. RBE, although measured quite simply in this way, depends on the complexities of how radiation of different qualities interact with different biological systems due to:

(1) The energy, depth, and mixture of Bragg peak or non-Bragg peak regions over a volume of interest.

(2) The increased local complexity, or clustering, of DNA damage with increasing LET (5).

(3) The increasing difficulty in repairing the more clustered damage, resulting in increased radiosensitivity and reduced fractionation sensitivity.

Some authors have developed relatively simple LET-RBE models for protons (6-8). For ion beams, there are several complex formulations that tentatively describe the relationship between LET and RBE (9-14), each with varying degrees of success, and have been used for clinical applications. These ion beam models are based on the fundamental interactions of particle physics with matter and contain multiple assumptions and input requirements, such as knowledge of particle trajectories relative to cells, cross sectional probabilities, the relative proportion of cell nucleus to cell volume for each cell, critical biological sub-volumes, repair capacities, and extrapolations with dose, etc. They all utilize long mathematical constructs which can be daunting to less mathematically gifted individuals. Whereas it is satisfying to build exploratory theoretical models in such a way, it is impossible to know these exact conditions within a real cancer and surrounding normal tissues. These various approaches have been used to predict ion beam RBE values for variable LET values for the irradiation of specific cell types (usually the V-79 cell derived from Chinese Hamsters), but with mixed results, although they are used routinely in clinical practice for carbon ion treatment planning. Only some authors have attempted an approach for normalizing the RBE differences between different ions, as in the work of Katz (9), who used the parameter $Z^{2} / \beta^{2}$ to calculate the radial distribution of dose (where $Z$ is nuclear charge and $\beta$ is the relativistic velocity).

What do we know with certainty about LET and RBE? Measured relationships between LET and RBE generally show increases with LET until a maximum value is achieved, followed by a decrease to RBE values just above unity. Also, there are important basic findings, shown by multiple authors (15-18), which are essential to incorporate into any model that adequately describes the change of RBE changing with LET. They are:

- The initial slope of RBE with LET is linear when plotted on linear scales (19).

- The LET value $\left(\mathrm{LET}_{\mathrm{U}}\right)$ which confers the maximum cell killing efficiency (at the turnover point) increases nonlinearly with the nuclear charge of a particle (the $Z$ number), which denotes the electrostatic positive charge of the particle nucleus. $\mathrm{LET}_{\mathrm{U}}$ values increase with $Z$, but smaller increments in $\operatorname{LET}_{\mathrm{U}}$ occur with increasing $Z$, which suggests a saturation effect. Ions with the smallest $Z$ values are consequently more efficient in increasing RBE per unit increase in LET, possibly because the energy released is more locally absorbed than is the case for higher $Z$ ions with larger event sizes and more energetic gamma emissions.

- The magnitude of the RBE is not only dependent on the particle type (or $Z$ ), but also depends on LET, dose (and so the surviving fraction of cells), and the cell type (and its ability to repair radiation damage).

- The magnitude of the RBE depends on cell type (seemingly regardless of the ion used), with cells that are intrinsically more radiosensitive (to very low LET radiations) having lower RBEs than their more radio-resistant counterparts.

- The RBE increases when the cell-surviving fraction is reduced (for lower doses), but the LET-RBE turnover point position remains constant. Thus, the overall symmetry is preserved.

- In terms of the linear quadratic model (LQ) of radiation effect, the $\alpha$ value increases with LET to a far greater extent than $\beta(20-22)$. The relative increase in $\alpha$ with LET is greatest for cells/tissues which have the lowest, most radioresistant, low LET $\alpha_{\mathrm{L}}$ values, with smaller increases in $\alpha$ for systems which have the most radiosensitive, highest intrinsic $\alpha_{\mathrm{L}}$ values, as will be shown later.

- These experimental findings apply to well-oxygenated cells, but are modified in radiologically hypoxic conditions (16), probably since the $\alpha$ parameter-related cell kill is not so influenced by oxygen as is the $\beta$ parameter-related cell kill.

This article considers how a much "simpler LET efficiency model" can estimate the LET-RBE relationships described above and their modification with dose, $Z$, and the two low LET intrinsic radiosensitivities $\alpha_{\mathrm{L}}$ and $\beta_{\mathrm{L}}$. These models require fewer input parameters and assumptions than do the far more complex models already referred to above. Such a model could be used to complement the other systems: in this sense, two predictions may carry more reliability if they are in close agreement.

\section{Materials and Methods}

\section{The Experimental Data}

There are relatively few published experiments that provide a reasonable estimate of LET turnover positions (LET $\mathrm{L}_{U}$ ) for clinically used particles. These include the data sets of Belli et al. (18), Barendsen et al. (15), Furusawa et al. (16), and Weyrather et al. (17). These experiments were not designed to accurately determine $\mathrm{LET}_{\mathrm{U}}$, but to show overall phenomena and to determine the range of RBE values. Inevitably, overall accuracy is further undermined by biological variation, use of different cellular assays in various laboratories, use of different LET interpretations and measurements over wide ranges with consequent use of a logarithmic scaled abscissa, which masks the uniform initial linear slope of the relationship. To obtain the best available estimate of $\mathrm{LET}_{\mathrm{U}}$, only the most unequivocal examples of maximum radiosensitivities, or $\mathrm{RBE}$, over a small range of LET near to $\mathrm{LET}_{\mathrm{U}}$, were used. Data where $\mathrm{LET}_{\mathrm{U}}$ could not be determined to reasonable accuracy, as in some of the HRG cellular data of Furusawa et al. (16) and in 
some carbon ion experiments, were excluded. The $\mathrm{LET}_{\mathrm{U}}$ values (keV/ $\mu \mathrm{m})$ so obtained were 30.5 (protons), 103.4 (helium), 208 (carbon), and 233 (neon). Although data exist for heavier ions such as silicon and argon, these do not provide a sufficiently accurate estimate (23).

For model fitting, the experimental studies of Todd (24), using a wide range of ions (deuterons, helium, lithium, boron, carbon, oxygen, nitrogen, neon, and argon), of Barendsen (deuterons and helium) (15), and of Weyrather et al. (carbon) (17) were used to test data against the modeled predictions.

The highest $\alpha$ radiosensitivity obtained $\left(\alpha_{U}\right)$, in the region of $\mathrm{LET}_{U}$, for each ion species, was plotted against the low LET (control) $\alpha_{\mathrm{L}}$ value from the same data. These values are shown in later graphical plots, and include variation due to the $\mathrm{LET}_{U}$ position uncertainty. The accuracy of the $\beta$ radiosensitivity parameter is less easy to determine for high LET radiations (compared with low LET radiations), for reasons discussed elsewhere $(8,22)$. It is known that $\beta$ increases to a lesser extent than $\alpha$ with LET. In order to maintain the observed constant position of LET $_{U}$ with increasing dose (and reduced surviving fraction), and the overall symmetry of the LET-RBE relationship, both $\alpha$ and $\beta$ must follow similar functions which rise to a maximum at $\mathrm{LET}_{\mathrm{U}}$. Otherwise, the overall symmetry of the LET-RBE curves with increasing dose would be broken: for example, if $\mathrm{LET}_{\mathrm{U}}$ would be different for $\alpha$ and $\beta$, the $\mathrm{LET}_{\mathrm{U}}$ would be observed to change with dose, which is not the case.

\section{Detailed Description of Model}

The turnover of RBE with LET, is a well-reported phenomenon often attributed to "overkill" or wasted local dose. This process can be interpreted as increasing efficiency of cell kill in the upward phase, followed by later inefficiency. In physics terms, the number of particle trajectories crossing a cell reduces by a reciprocal function of increasing LET after the turnover point. This is necessary in order to maintain the same overall dose to a wider volume with increasing LET. At the same time, increasing LET produces greater clustering of dose deposition, but overclustering will not necessarily lead to enhanced biological effects. In bio-physical terms, increasing LET must, initially, enhance the intrinsic radiosensitivity parameters, the increment in $\alpha$ far exceeding that in $\beta$ (25). This is because a greater proportion of more clustered damage is non-repairable by the non-homologous end joining process, although the repair of sub-lethal damage (within the more sparsely clustered damage regions) continues, although probably with lower fidelity, and even the recombination repair mechanism may also be overwhelmed by increasingly complex lesions affecting the same sites on sister chromatids.

Ionizing radiation damage in biological systems causes a hierarchy of effects: the most commonly occurring DNA base change and single strand breaks are followed by less frequent double strand breaks, nearly all of which are repaired in the case of low LET radiation. An excess of a mixture of these forms of damage in a locality of a chromosome can lead to a chromosome break, certain types of which inevitably confers lethality. Thus, the essential lesion is the "lethal" form of chromosome break (LCB) for most forms of radiation cell death at clinical doses. The local deposition of energy that results in the maximum probability of a single
LCB must represent the maximum efficiency of the system, since further energy deposition and greater DNA and chromosomal structural change in the same locality will result in no extra effect; in fact any dose deposited in excess of that required to achieve a LCB will be "wasted dose," representing inefficiency, and is often referred to as the overkill effect.

On a local basis, with LET defined as being the energy deposited over a $1 \mu \mathrm{m}$ section of track, this distance is appropriate for chromosomal radiation effects since it is roughly the width of a single chromosome.

\section{Relationship between $Z$ and LET $_{U}$}

The position of the turnover point can be estimated for different $Z$ values. It is apparent from publications quoted above (15-18) that $\mathrm{LET}_{\mathrm{U}}$ increases with $Z$, but the effect appears to saturate (i.e., further increase in LET have diminishing returns as far as the $\mathrm{LET}_{\mathrm{U}}$ value is concerned). The Betha-Bloch equation for estimating the rate of energy loss with distance $(x)$ traversed $(\mathrm{dE} / \mathrm{dx})$, which represents LET, contains a $Z^{2}$ term in the numerator usually reflecting the charge of a fully electron stripped ion or proton. Larger $Z$ values will also be associated with larger mass numbers and greater momentum with larger event volumes due to more complex nuclear collisions and energetic $\gamma$ ray emissions. Beyond the necessary critical dimension (be this radial or linear as a surrogate), biological killing efficiency will not increase if the event size becomes too large and physically beyond the individual chromosome. So, a saturation effect is to be expected. The smallest values of $Z=1$ for a proton effectively reduces $\mathrm{dE} / \mathrm{dx}$, but the proton $\mathrm{LET}_{\mathrm{U}}$ is only $30.5 \mathrm{keV} / \mu \mathrm{m}$, suggesting that lighter charged particles exert more localized effects (caused by short range low energy secondary electrons). In this respect, the proton is more efficient at causing an increment in RBE with LET (but proton LET values are quite small, e.g., a LET of only $1-8 \mathrm{keV} / \mu \mathrm{m}$ in typical clinical exposures $(26,27)$ when using scanned proton beams may cause RBEs as high as 1.8 or more (8)].

The application of a simple differential equation can represent this process. Let us assume that $Z$ is a continuous variable and if the initial rate of change in $\mathrm{LET}_{\mathrm{U}}$ with $Z$ is $S$ and that this value then decreases in proportion to $\mathrm{LET}_{U}$ itself, representing a saturation effect controlled by the constant $k$, so that

$$
\frac{d \mathrm{LET}_{\mathrm{U}}}{d Z}=S-k \cdot \mathrm{LET}_{\mathrm{U}}
$$

which by integration of both sides and rearrangement leads to

$$
\operatorname{LET}_{\mathrm{U}}=S / k(1-\operatorname{Exp}[-k(Z)]
$$

where $S / k$ represents the maximum possible value of $\mathrm{LET}_{\mathrm{U}}$.

Equation 2 can be normalized to the proton $(Z=1) \operatorname{LET}_{U}$ of $30.5 \mathrm{keV} / \mu \mathrm{m}$ found by Belli et al. (16), so that for any $Z$ a term $Z-1$ is used such that:

$$
\mathrm{LET}_{\mathrm{U}}=30.5+S / k(1-\operatorname{Exp}[-k(Z-1)])
$$

This equation is used for data fitting purposes. 


\section{Changes in Radiosensitivities with LET}

By increasing LET gradually, from the control low LET value of say clinical 4-6 MV photons (X-rays), we obtain small increases in the probability of additional LCBs; the energy deposition becomes maximally efficient (let this be represented by $100 \%$ efficiency for normalization purposes), and at higher LET values beyond $\mathrm{LET}_{\mathrm{U}}$, the efficiency is reduced below $100 \%$ because of excess local energy deposition.

The separate relationship between $\alpha_{\mathrm{L}}$ (the low LET control $\alpha$ value) and $\alpha_{U}$ (the value of $\alpha$ at the turnover point where $\mathrm{LET}=\mathrm{LET}_{\mathrm{U}}$ ) also exhibits saturation effects. In other words, the increment in $\alpha$ with LET show diminishing returns, since the lowermost $\alpha_{L}$ values have the highest gain in $\alpha$. This effect is found with fast neutrons and with charged particle data, as shown in the Section "Results" below.

For an initial slope of $A$ and a rate constant $j$, the rate of change of $\alpha_{U}$ with $\alpha_{L}$ will fall in proportion to $\alpha_{U}$, so that

$$
\frac{d \alpha_{\mathrm{U}}}{d \alpha_{\mathrm{L}}}=A-j \cdot \alpha_{\mathrm{U}}
$$

which leads after integration to:

$$
\alpha_{\mathrm{U}}=A / j\left(1-\operatorname{Exp}\left[-j \alpha_{\mathrm{L}}\right]\right) \text {. }
$$

The $\beta$ parameter can either be modeled in a similar way but with smaller overall changes, or to simplify matters for tentative modeling purposes, it could be assumed to be invariant at low doses where $\beta$-related cell kill is small. The data of Weyrather et al. (17) show that $\beta$ values rises from a control value of $0.026 \mathrm{~Gy}^{-2}$ to a maximum of $0.044 \mathrm{~Gy}^{-2}$ in $\mathrm{V}-79$ cells, and likewise from 0.02 to $0.42 \mathrm{~Gy}^{-2}$ for the $\mathrm{CHO}$ cells $\left(\alpha / \beta\right.$ value of $0.192 \mathrm{~Gy}^{-2}$ in one instance must be artifactual due to the fitting program), i.e., by up to a factor of around two, which is small compared to the maximum increments in $\alpha$ with LET of around 10. There is more abundant data for $64 \mathrm{MV}$ fast neutrons where $\beta$ undoubtedly increases (22). Although such neutron experiments will probably underestimate the maximum possible rise in $\alpha$ and $\beta$, since the neutron LET spectrum (and its average value) may not necessarily be close to the $\mathrm{LET}_{U}$ for an ionic beam. Nevertheless, further analysis of these data, which compare neutrons with megavoltage $\mathrm{X}$-rays show fits of $\beta_{\text {neu }}=1.54 \beta_{x-\text { ray }}$ or $\beta_{\text {neu }} 0.097$ [ $1-\operatorname{Exp}$ $\left(23.6 \beta_{x-\text { ray }}\right)$, as will be shown below]. The experimental variation in such data is considerable and the two fitted equations were obtained after elimination of: repair deficient cells (where $\alpha>0.6 \mathrm{~Gy}^{-1}$ ), or where neutron $\beta$ values close to zero, or if the increment in $\beta$ exceeded that in $\alpha$ (suggesting experimental artifact). It should be noted that $\alpha_{U}$ and $\beta_{U}$ values will be higher than the maximum values obtained for fast ( $64 \mathrm{MV})$ neutrons, and so the neutron data cannot be used directly to determine RBE changes for ion beam data.

A similar "saturation" function, is used to link $\beta_{\mathrm{L}}$ with $\beta_{\mathrm{U}}$, as given elsewhere (8):

$$
\beta_{\mathrm{U}}=(R / u) \cdot\left(1-\operatorname{Exp}\left[-u \beta_{\mathrm{L}}\right]\right) .
$$

Where $R=2.5$ and $u=25$, which provides a modest increase in $b$, and is compatible with the limited data discussed already, and with a maximum ceiling value of $0.1 \mathrm{~Gy}^{-2}$ for $\beta_{\mathrm{U}}$.

\section{Obtaining $\alpha_{H}$ and $\beta_{H}$ values}

In simple mathematical terms, a discontinuous or biphasic (efficiency followed by inefficiency) model can be used, where for LET values up to that of $\operatorname{LET}_{U}$, increasing efficiency is represented as a linear simple proportional relationship, as used by Wilkens and Oelfke for protons (6), and where the $\alpha$ value at any LET higher than the control and lower than the turnover value will be

$$
\alpha_{\mathrm{H}}=\alpha_{\mathrm{L}}+\frac{\mathrm{LET}_{\mathrm{x}}-\mathrm{LET}_{\mathrm{C}}}{\mathrm{LET}_{\mathrm{U}}-\mathrm{LET}_{\mathrm{C}}} \cdot\left(\alpha_{\mathrm{U}}-\alpha_{\mathrm{L}}\right)
$$

where $\alpha_{H}$ is the $\alpha$ value at any particular LET value $\left(\operatorname{LET}_{\mathrm{x}}\right)$ between the control and ultimate value of $\mathrm{LET}_{\mathrm{x}}$ [which represents any LET value between the control value of $\operatorname{LET}_{C}$ (where $\alpha$ is $\alpha_{\mathrm{L}}$ )] and $\mathrm{LET}_{U}$, where the maximum $\alpha$ of $\alpha_{U}$ occurs.

For the initial linear portion of the relationship, there will be a uniform gradient of

$$
\frac{\alpha_{U}-\alpha_{\mathrm{L}}}{\mathrm{LET}_{\mathrm{U}}-\mathrm{LET}_{\mathrm{C}}}
$$

between the value of $\mathrm{LET}_{\mathrm{C}}$ and $\mathrm{LET}_{\mathrm{U}}$, which fulfills the requirement for linearity in this range of LET. It follows that, for example, if $\mathrm{LET}_{\mathrm{C}}$ and $\mathrm{LET}_{\mathrm{U}}$ are 1.2 and $120 \mathrm{KeV} / \mu \mathrm{m}$ respectively, with $\alpha_{\mathrm{L}}$ and $\alpha_{U}$ of say 0.3 and $1.3 \mathrm{~Gy}^{-1}$, then for a $\operatorname{LET}_{\mathrm{x}}$ value of 60 , the process is only $(1.3-0.3) /(120-1.2) \times(60-1.2)$, which is close to being $50 \%$ efficient, and for a $\mathrm{LET}_{\mathrm{x}}$ of 90 , the efficiency will be $(1.3-0.3) /(120-1.2) \times(90-1.2)$, which is close to $75 \%$ efficiency.

In this way, the efficiency of cell kill per unit dose will increase linearly with LET, leading up to maximum efficiency (defined as $100 \%)$ at $\mathrm{LET}_{\mathrm{U}}$.

For values of LET beyond the turnover point (where LET $>\mathrm{LET}_{\mathrm{U}}$ ), the additional energy transferred does not contribute to extra lethality, but is wasted. That is, the excess energy $\left(\mathrm{LET}_{\mathrm{x}}-\mathrm{LET}_{\mathrm{U}}\right)$ beyond the optimal released energy is wasted. Consequently, inefficiency, expressed in energy terms by $\left(\mathrm{LET}_{\mathrm{x}}-\mathrm{LET}_{\mathrm{U}}\right) / \mathrm{LET}_{\mathrm{U}}$ increases. To express this in terms of efficiency, the relationship of: $\%$ efficiency $=100-\%$ inefficiency is used, and the $\alpha_{H}$ value is then scaled between $\alpha_{U}$ and $\alpha_{C}$.

Accordingly, the equation for $\alpha_{H}$ for LET $>$ LET $_{U}$ then changes to be:

$$
\alpha_{\mathrm{H}}=\alpha_{\mathrm{L}}+\left(1-\frac{\mathrm{LET}_{\mathrm{x}}-\mathrm{LET}_{\mathrm{U}}}{\mathrm{LET}_{\mathrm{U}}}\right) \cdot\left(\alpha_{\mathrm{U}}-\alpha_{\mathrm{L}}\right)
$$

which effectively expresses the reduction in $\alpha$ with increasing LET. In this way, if $\mathrm{LET}_{\mathrm{x}}$ is 180 and $\mathrm{LET}_{U}$ is 120 , the value of $\alpha_{U}$ at the turnover point of $100 \%$ efficiency will fall to $1-(180-120) / 180$, which provides around $67 \%$ efficiency. For a $\mathrm{LET}_{\mathrm{x}}$ of 240 , we obtain $1-(240-120) / 240$, which is $50 \%$ efficient. These efficiencies are of course relative to a normalized value of $100 \%$ at the turnover point.

Similar equations are used to provide $\beta_{\mathrm{H}}$, by proportionate scaling between $\beta_{\mathrm{L}}$ and $\beta_{\mathrm{U}}$. These are obtained by simply replacement of $\alpha_{\mathrm{L}}, \alpha_{\mathrm{H}}$, and $\alpha_{\mathrm{U}}$ by $\beta_{\mathrm{L}}, \beta_{\mathrm{H}}$, and $\beta_{\mathrm{U}}$ respectively in Eqs (7) and (9).

\section{Reduction of RBE with Dose}

The reduction in RBE with reduced surviving fraction and increasing dose is obtained by the solution of the following isoeffect equation for high and low LET radiations at a dose $d_{\mathrm{L}}$ and $d_{\mathrm{H}}$, for low and high LET respectively:

$$
\alpha_{\mathrm{L}} d_{\mathrm{L}}+\beta_{\mathrm{L}} d_{\mathrm{L}}^{2}=\alpha_{\mathrm{H}} d_{\mathrm{H}}+\beta_{\mathrm{H}} d_{\mathrm{H}}^{2}
$$


The solution for $d_{\mathrm{L}}$ is then divided $d_{\mathrm{H}}$ to provide the RBE, as shown in other publications $(8,24,25)$.

For clinical iso-effect calculations, the solution of the following biological effective dose (BED) equations are used for the low and high LET:

$$
n d_{\mathrm{L}}\left(1+\frac{d_{\mathrm{L}}}{\left(\frac{\alpha}{\beta}\right)_{\mathrm{L}}}\right)=m d_{\mathrm{H}}\left(\mathrm{RBE}_{\mathrm{Max}}+\frac{\mathrm{RBE}_{\mathrm{Min}}^{2} \cdot d_{\mathrm{H}}}{\left(\frac{\alpha}{\beta}\right)_{\mathrm{L}}}\right),
$$

where $n$ and $m$ are the respective numbers of fractions for the low and high LET.

The RBE parameters are replaced by LET (and the new parameters given in the sequence of equations described above) and then solved for $d_{\mathrm{H}}$. Total doses to provide the same BED can then be calculated for different numbers of fractions.

Computer programs using Mathematica (Champagne, IL, USA) software were constructed using the above equations.

\section{Results}

The relationship between $Z$ and $\mathrm{LET}_{\mathrm{U}}$ shown in Figure 1, using pooled data for proton, helium, carbon, and neon ions (13-16), were fitted by Eq. (3).

The Clatterbridge fast neutron data (21), show the relationship between $\alpha_{\mathrm{L}}$ (for values up to $0.8 \mathrm{~Gy}^{-1}$ ) and $\alpha_{\mathrm{H}}$, and between $\beta_{\mathrm{L}}$ and $\beta_{\mathrm{H}}$, are shown in Figures $2 \mathbf{A}, \mathbf{B}$, respectively. In each case, the linear and non-linear fits are not significantly different $(p>0.05)$, although the residuals are smallest for the non-linear equations, which also have the advantage of not extrapolating to infinitely high radiosensitivity values.

The relationship between $\alpha_{L}$ and $\alpha_{U}$ for various ions are shown in Figure 3, fitted to data from the literature [with data where negative $\beta$ values obtained excluded]. The fitted equation is shown in the figure, but also with a least squares fit for a linear nointercept relationship of $\alpha_{\mathrm{U}}=6.47 \alpha_{\mathrm{L}}\left(p<0.001, R^{2}=0.899\right)$ for $\alpha_{\mathrm{L}}$ values less than $0.35 \mathrm{~Gy}^{-1}$, the more radio-resistant part of the radiosensitivity spectrum.

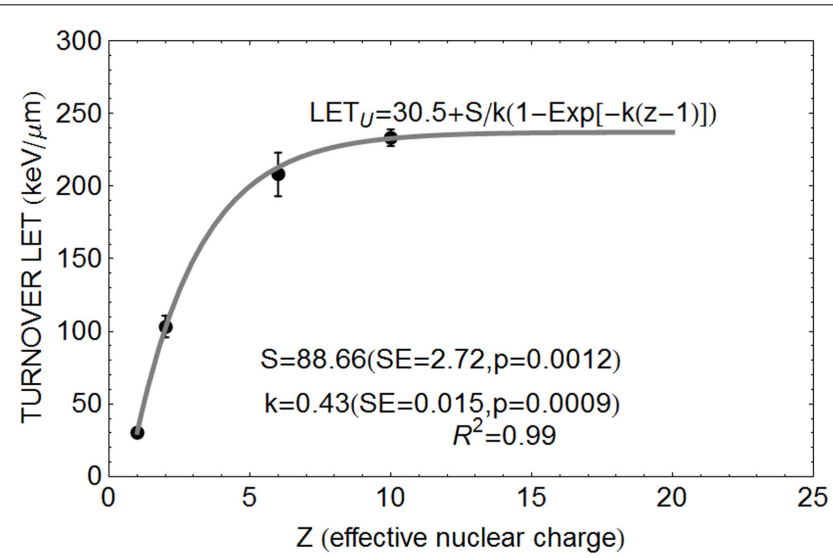

FIGURE 1 | Data points for relationship between $Z$ and turnover point LET value LETu with fitted parameter values based on Eq. (3).

\section{Fits to Experimental RBE Data Sets}

The model is superimposed to the experimental data sets, using different cell lines, of Barendsen (Figure 4) and Weyrather et al. (Figures 5A,B) and Todd (Figures 6 and 7).

The data of Barendsen used mono-energetic deuterium or helium (alpha) particles in one human cell type, with highly symmetrical curves which turnover at around $110 \mathrm{keV} / \mu \mathrm{m}$. In this case (see Figure 4), the model fits the data reasonably well at all levels of surviving fraction. However, since this data set exists as plotted graphical surviving fraction results without access to the original data, there is inevitable uncertainty in assessing the low
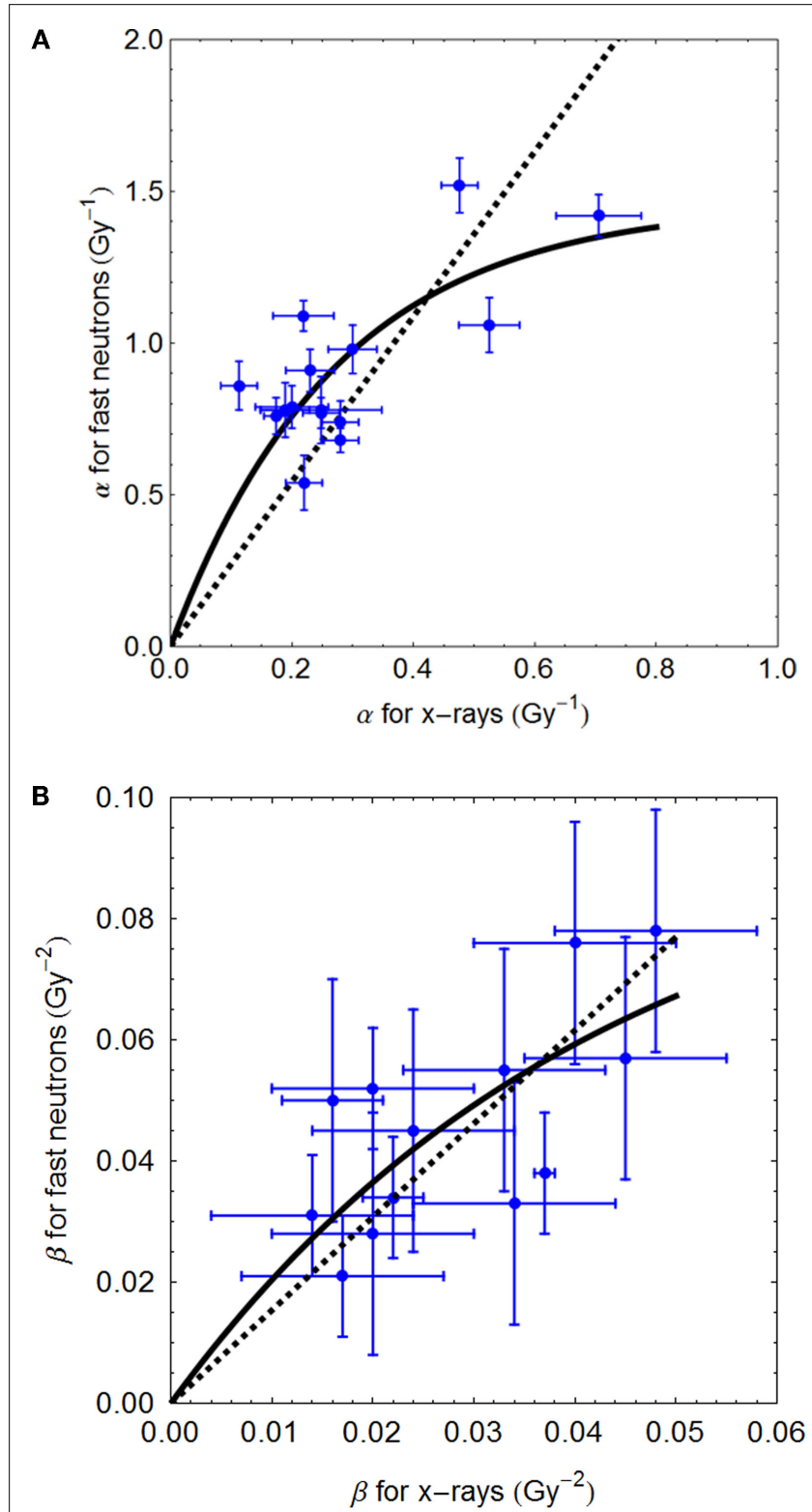

FIGURE 2 | (A,B) Sixty-four megavolt fast neutron relationships between low and high LET radiosensitivity parameters. Linear no-intercept and non-linear least squares fits are respectively: (A) $\alpha_{H}=2.72 \alpha_{\mathrm{L}}$ and $\alpha_{H}=5.37 / 3.68$ $\left(1-e^{-3.68 \alpha \mathrm{L}}\right) ;$ (B) $\beta_{\mathrm{H}}=1.57 \cdot \beta_{\mathrm{L}}$ and $2.29 / 23.57\left(1-\mathrm{e}^{-23.57 \beta \mathrm{L}}\right)$ using Mathematica software. 


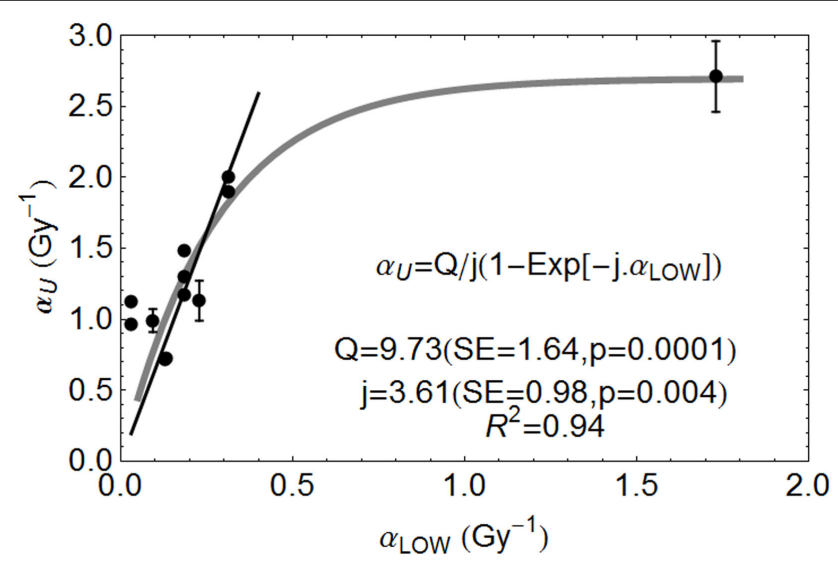

FIGURE 3 | Ion beam relationships between radiosensitivity parameters at low and high LET at the turnover point ( $\alpha_{H}$ is here $\left.\alpha_{U}\right)$ and fitted by the parameters shown, using Mathematica software. Error bars are not available for all data used. Reproduced with permission from Ref. (8).

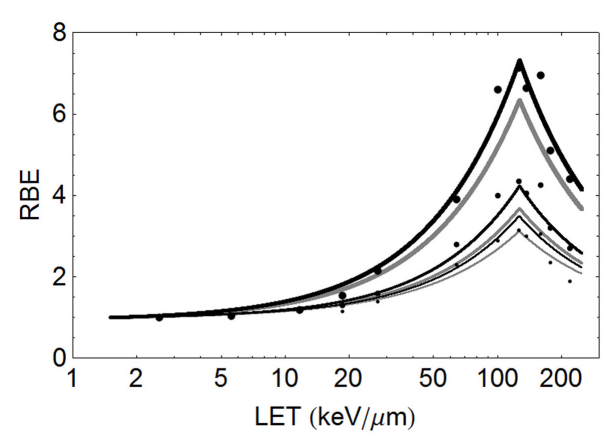

FIGURE 4 | Mono-energetic alpha particle data of Barendsen (with large points indicating $50 \%$ survival, medium sized points $10 \%$ survival, and the smallest points $5 \%$ survival, using the proposed model to provide fit lines, with black indicating use of parameters $\alpha_{L}=0.16 \mathrm{~Gy}^{-1}, \alpha_{U}=1.31 \mathrm{~Gy}^{-1}, \beta_{L}=0.046 \mathrm{~Gy}^{-2}, \beta_{U}=0.15 \mathrm{~Gy}^{-2}$ and gray using parameters $\alpha_{\mathrm{L}}=0.15 \mathrm{~Gy}^{-1}, \alpha_{U}=1.35 \mathrm{~Gy}^{-1}$, $\boldsymbol{\beta}_{\mathrm{L}}=0.03 \mathrm{~Gy}^{-2}, \boldsymbol{\beta}_{\mathrm{U}}=0.08 \mathrm{~Gy}^{-2}$. The thickest lines are for $50 \%$ survival, medium lines for $10 \%$ survival, and thinnest lines for $5 \%$ survival.

and high LET $\alpha$ and $\beta$ values, which make the RBE determination even more difficult. The plot was obtained by assessment using $\alpha_{\mathrm{L}}=0.16, \alpha_{U}=1.31, \beta_{\mathrm{L}}=0.046, \beta_{\mathrm{U}}=0.15$ obtained by crude measurements of survival curves and RBE plots, each on a logarithmic and linear scales, drawn by artists and which contain displacements of many data points for convenience of display, but the data set is better fitted by $\alpha_{\mathrm{L}}=0.15, \alpha_{U}=1.35, \beta_{\mathrm{L}}=0.03$, $\beta_{U}=0.08$, as shown in Figure 4. The Barendsen data set suffers from retrospective inaccuracies in estimating parameters from diagrams in publications rather than use of the raw data, but the graphic shows the sensitivity of the model to the input parameters.

The critical dependency of each RBE limit on the ratio of $\alpha$ and $\beta$ at low and high LET respectively, demonstrates the importance of obtaining the most accurate possible data, rather than depending on published material which does not contain precise surviving fraction outcomes. The Barendsen data set also suggests a higher value of $\mathrm{LET}_{\mathrm{U}}$ for alpha particles than obtained above
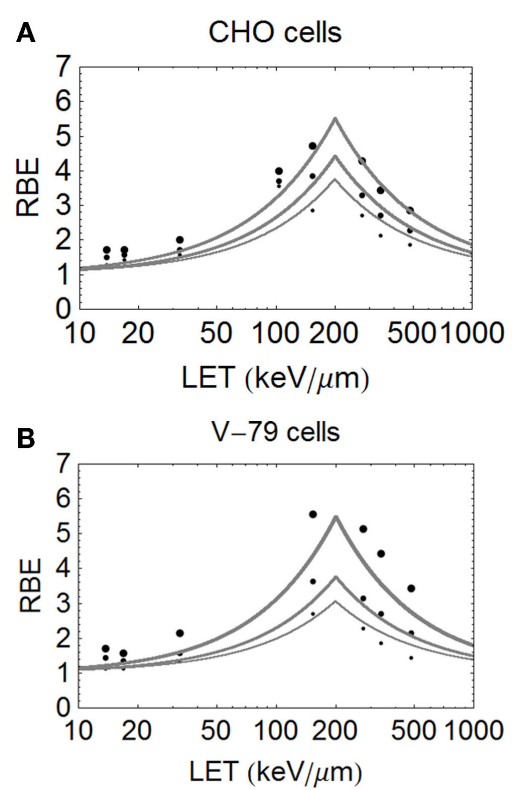

FIGURE 5 | (A,B) Model fitted data of Weyrether et al. for $\mathrm{C}$ ions for three different cell lines and doses, coded in the same way as for Figure $\mathbf{4}$ with respect to line thickness and surviving fraction (A) for $\mathrm{CHO}$ cells and (B) for $\mathrm{V}-79$ cells.

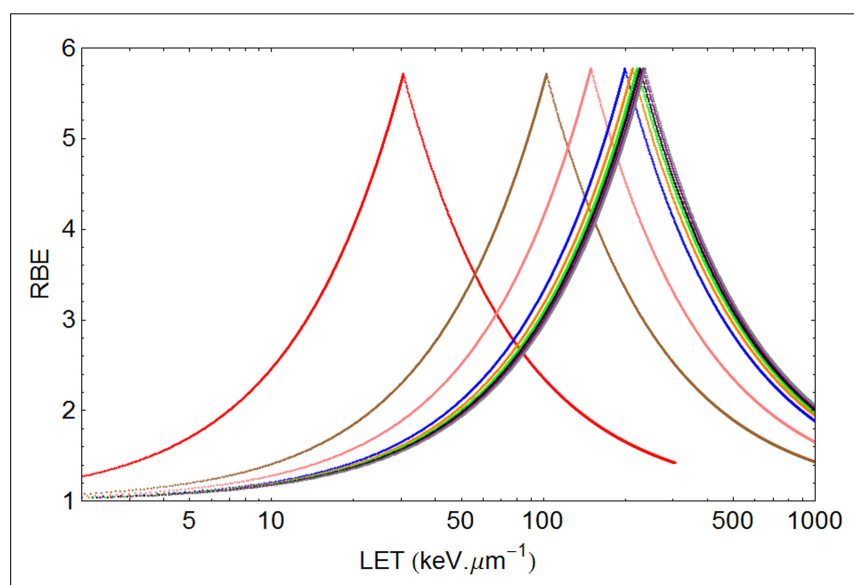

FIGURE 6 | Graphical displays of RBE and LET with unique turnover point positions for multi-ion data of Todd, assuming $\alpha=0.14 \mathrm{~Gy}^{-1}$ and $\beta=0.05 \mathrm{~Gy}^{-2}$ for a dose of $1.5 \mathrm{~Gy}$. From left to right the ionic elements are shown as follows, with color code and LET $($ rounded to nearest integer for values over 100) in parentheses: Deuterium (red, 30.5), Helium (brown, 103), Lithium (pink, 150), Boron (blue, 200), Carbon (orange, 213), Nitrogen (green, 221), Oxygen (Black, 227), Neon (purple, 232), and Argon (gray, 237).

using the formula based on $Z$ in pooled data, at around 127 instead of $103 \mathrm{keV} / \mu \mathrm{m}$; also the $\alpha_{U}$ is predicted to be $1.18 \mathrm{~Gy}^{-1}$ by Eq. (3). This illustrates the uniqueness of each data set and the distorting effect of pooling of data from different laboratories using different cell systems etc.

The important carbon ion data of Weyrather et al. (17), from GSI, which covers a broader range of LET values, shows an apparently constant turnover point for different cell types and surviving fractions (Figures $\mathbf{5 A}, \mathbf{B})$. The data are published with the LQ 


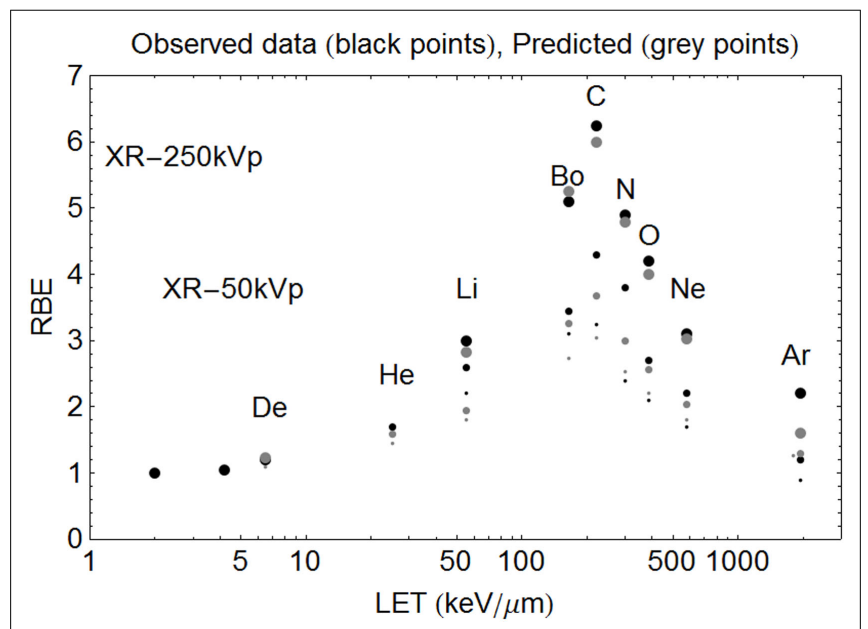

FIGURE 7 | Model predictions shown with data of Todd using the new $Z$-based model to determine LET $U$ and $\alpha_{U}, \beta_{U}$ respectively, with LQ model correction for dose. The largest sized points are for $\mathrm{SF}=50 \%$, the intermediate sized points are for $\mathrm{SF}=10 \%$, and the smallest sized points are for $\mathrm{SF}=1 \%$. The ions used and their $\mathrm{LET}(\mathrm{keV} / \mu \mathrm{m})$ values are given respectively in parentheses. Deuterium (6.5), Helium (25), Lithium (55), Boron (165), Carbon (220), Nitrogen (300), Oxygen (385), Neon (580), and Argon (1940). Observed RBE data are printed as black points, with estimated RBE values as gray points. Starting on the left hand side the first two black points are for 250 and $50 \mathrm{kV} \mathrm{X}$-rays respectively, followed by deuterons etc.

radiosensitivities, although the ions have a small variation in their LET spectrum (with a maximum spread of less than 5\% for the highest LET values which reduces further with decreasing LET). So, it is unlikely that energy and LET spread contribute to the deviations from the modeled curves seen at lower LET values. The RBE values found at low LET values seem higher than expected, possibly due to biological sample variation, especially since irradiations were performed using two different accelerator systems (for LET values above and below $100 \mathrm{keV} / \mu \mathrm{m}$ ) in different laboratories and presumably at different times. These data, although very informative, inevitably contain greater heterogeneity than the data of Barendsen, and the data are less well fitted. Another more stochastic approach is to use a Poisson function, which will be presented in a further publication.

In the case of Todd's multi-ion data (24), a range of different mono-energetic ions were used (protons, deuterium, helium, lithium, boron, carbon, nitrogen, oxygen, neon, and argon), which implies that there will be at least nine different curves, one for each $Z$ value, and each with unique turnover points. Such heterogeneous data were fitted surprisingly well by allocating a unique turnover point for each ion species, before estimation of the RBE, as shown in Figure 6, followed by the RBE estimations for each ionic species in Figure 7.

\section{Clinical Radiobiology}

It is possible to tentatively assess changes in total dose required for different fractionation schedules using protons, helium, and carbon ions, as shown in Figures 8A-C. The variations in LET are representative of the wide expected clinical ranges for nonBragg peak regions and spread out Bragg peaks of different sizes and for scanned beams. It should be noted that the changes in total

\section{A protons}

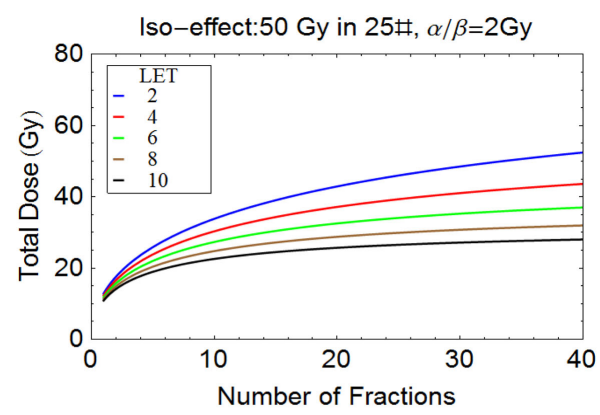

B Helium ions

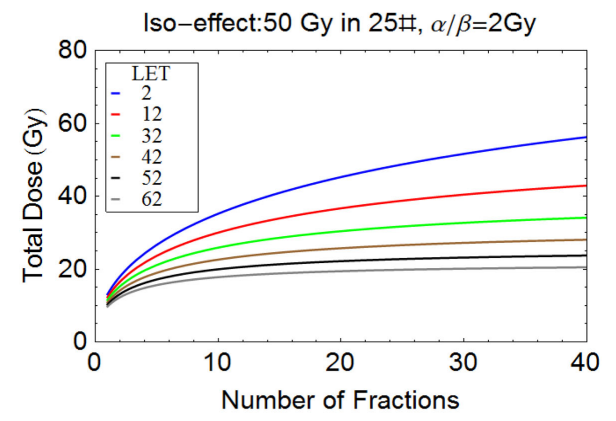

C Carbon ions

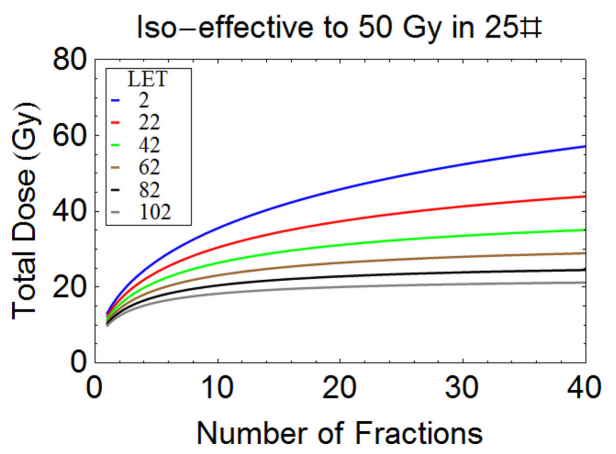

FIGURE 8 | (A-C) Plots of total iso-effective dose versus number of fractions for the given iso-effect and $\alpha / \beta$ ratio. (A) Protons, (B) Helium ions, (C) Carbon ions.

dose required with number of fractions (and consequently dose per fraction) are remarkably similar for the respective LET ranges used. This indicates the importance of LET mapping as well as dose mapping in the clinic, since RBEs and consequently changes in total dose with fractionation can be the same for a wide range of ions, as determined by their $Z$ value and LET.

\section{Discussion}

Simple differential equations which model saturation effects are commonly used in the physical sciences and in biology, with notable examples in pharmacokinetics. Saturation in the radiation context applies to the relationship between the effective event size and the bio-target. Maximum efficiency represents the maximum 
cell killing effect caused by locally absorbed energy, which differs from the energy released, some of which may be wasted by causing more local damage than is necessary to cause lethality, or is dissipated over a wider than necessary critical volume.

The new model offers a relatively simpler semi-empirical mathematical method for assessing changes in RBE with LET than has previously been available and provides a second order approximation. It can be more easily understood and used by clinicians, biologists, and others, without recourse to more complex mathematics. Also, the two saturation-based assumptions made, in comparison, are fewer than the assumptions required in other RBE models. For highly controlled and relatively homogenous data sets, this deterministic approach provides reasonable estimates of RBE. For protons, a variant of this approach has been published recently (8). The model depends on the assumptions of the LQ model where $\alpha$ and $\beta$ are high level parameters, being ultimate coefficients of radiation induced cell death, rather than basic components of radiation effect such as DNA strand breaks etc.

The model is not intended to supplant existing models of RBE, but to be complementary. It would be highly advantageous in clinical practice if more than one model could be used, with clinical decisions allowed to proceed if at least two are in reasonable agreement. Thus, the LEM, MKM, and variants of the Katz models should continue to be used, and compared with the new model.

Improved input data would undoubtedly further improve the accuracy of the model. Rather than attempt to fit historical data, which are limited in terms of accurate determination of "maximum efficiency" turnover points, it would be better to conduct rigorous experiments to test hypotheses connected with the above models, such as the relationship of the initial slope to more precise estimates of the turnover point position $\left(\mathrm{LET}_{\mathrm{U}}\right)$ in different ions. This also requires a further stochastic interpretation necessary to match a range of LET values as would be encountered in many clinical beams. There is ample scope for research in this respect.

Some authors have emphasized the inverse association between low LET $\alpha / \beta$ and the final $\operatorname{RBE}(7,13)$. This follows since $\alpha / \beta$ reflects repair capacity and intrinsic radiosensitivities, and is valid more at low doses. From the definitions of $\mathrm{RBE}_{\max }$ and $\mathrm{RBE}_{\min }$, it is easy to show that the former will be inversely related to related to $(\alpha / \beta)_{\mathrm{L}}$, but the latter directly proportional to the square root of $(\alpha / \beta)_{L}(21,28)$. The former assumption can be used for low dose per fraction treatments, where $\mathrm{RBE}_{\max }$ dominates the RBE. The need to include changes in $\beta$ with LET is necessary for estimations of RBE at higher doses, and where $\alpha / \beta$ is small as in human late tissue effects. The new model also preserves the overall symmetry of the curves at increasing dose. Accurate estimation of $\beta$ from cell survival curves, especially when $\alpha$ values are large, are notoriously difficult to achieve. Our knowledge of how $\beta$ changes with increasing LET is less well documented than for the larger and easier to measure changes in $\alpha$ with increasing LET. Only by meticulously conducted large scaled experiments, with greater than usual numbers of cell survival experiments, can these parameters be estimated to greater and sufficient accuracy.

Since neutrons are uncharged, they do not fall easily into this model, although the main products of neutron interactions such as recoil protons and other ions do, such that a spectrum of LET values will result, which in principle could be translated into RBE using the modeling described in this report. Again this would require further specific study.

There is considerable scope for the application of simpler RBE predictive models. Ideally prospective experiments should be performed with specific attention to LET-RBE turnover point position for different ions, the initial slope of the increment in RBE and the maximum value of $\alpha$ and $\beta$ relative to their low LET values. These need to be determined for extensive in vitro libraries of human cell lines and, if confirmed, extended to more complex in vivo experiments. A single international center would be ideal for this purpose, as has already been proposed at CERN $(29,30)$. There, it might be possible to create a new extensive data base for LET-RBE relationships, and to re-confirm or refute the basic $\mathrm{RBE}$ principles listed on p. 3. Of special concern are the slopes of the relationship, and improved accuracy for key $\mathrm{LET}_{\mathrm{U}}$ parameter, using multiple ion species in an appropriate panel of human cell lines, and to a much higher degree of accuracy than previously obtained. In this way, the data shown in Figures $\mathbf{1}$ and $\mathbf{3}$ could be enhanced by experiments on multiple ion species. Also, the results of all available models should be compared in such a single laboratory.

Such a project must be regarded as "essential science" for informing clinical practice, so that the best outcomes from particle therapy may in the future be fully, rather than partially, realized. Many practical enigmas remain within particle therapy $(8,31)$.

It is noteworthy for medical scientists to realize that in the first 6 weeks of the experiments that lead to the discovery of the theoretically predicted Higgs Boson, the entire laws of particle physics were not only re-confirmed, but to a much higher level of accuracy than previously achieved. Similar goals must be attempted in radiobiology, although over a longer time frame. This would provide the tools for greater predictive accuracy to particle radiotherapy, to improve its efficacy, as well as provide enhanced knowledge for human radiation protection, including astrobiology.

\section{Acknowledgments}

To colleagues who have inspired me over many years to seek solutions to high LET problems, especially Roger Dale, Gillies McKenna, John Hopewell, Oliver Scott, Jack Fowler, Mark Hill, Herman Suit, Peter O'Neil, and Dudley Goodhead. I am grateful to CERN, Geneva for a Visiting Scientist award during 2014, and to the Director General for the award of Guest Professor 2015-2016. Also, to the generosity of the Principal and Fellows of Brasenose College, Oxford. BJ has been an investigator on several UK Research Council and EU FP-7 funded grants concerned with particle therapy, including ENVISION (241851), ENTERVISION (264552), and ULICE (228436). 


\section{References}

1. Durante M, Loeffler JS. Charged particles in radiation oncology. Nat Rev Clin Oncol (2010) 7:37-43. doi:10.1038/nrclinonc.2009.183

2. Jones $B$. The potential advantages of charged particle radiotherapy using protons or light ions. Clin Oncol (2008) 20:555-63. doi:10.1016/j.clon.2008.02.012

3. Kamada T, Tsujii H, Blakely EA, Debus J, De Neve W, Durante M, et al. Carbon ion radiotherapy in Japan: an assessment of 20 years of clinical experience. Lancet Oncol (2015) 16(2):e93-100. doi:10.1016/S1470-2045(14)70412-7

4. Combs SE, Debus J. Treatment with heavy charged particles: systematic review of clinical data and current clinical (comparative) trials. Acta Oncol (2013) 52(7):1272-86. doi:10.3109/0284186X.2013.818254

5. Anderson RM, Stevens DL, Sumption ND, Townsend KM, Goodhead DT, Hill MA. Effect of linear energy transfer (LET) on the complexity of alpha-particleinduced chromosome aberrations in human CD34+ cells. Radiat Res (2007) 167(5):541-50. doi:10.1667/RR0813.1

6. Wilkens JJ, Oelfke U. A phenomenological model for the relative biological effectiveness in therapeutic proton beams. Phys Med Biol (2004) 49(13):2811-25. doi:10.1088/0031-9155/49/13/004

7. Carabe A, Moteabbed M, Depauw N, Schuemann J, Paganetti H. Range uncertainty in proton therapy due to variable biological effectiveness. Phys Med Biol (2012) 57:1159-72. doi:10.1088/0031-9155/57/5/1159

8. Jones B. Towards achieving the full clinical potential of proton therapy by inclusion of LET and RBE models. Cancers (Basel) (2015) 7(1):460-80. doi:10. 3390/cancers7010460

9. Katz R, Ackerson B, Homoyooufar M, Sharma SC. Inactivation of cells by heavy ion bombardment. Radiat Res (1971) 47:402-25. doi:10.2307/3573247

10. Elsässer T, Krämer M, Scholz M. Accuracy of the local effect model for the prediction of biologic effects of carbon ion beams in vitro and in vivo. Int $J$ Radiat Oncol Biol Phys (2008) 71(3):866-72. doi:10.1016/j.ijrobp.2008.02.037

11. Kase Y, Kanai T, Matsufuji N, Furusawa Y, Elsässer T, Scholz M. Biophysical calculation of cell survival probabilities using amorphous track structure models for heavy-ion irradiation. Phys Med Biol (2008) 53(1):37-59. doi:10.1088/ 0031-9155/53/1/003

12. Grün R, Friedrich T, Krämer M, Zink K, Durante M, Engenhart-Cabillic R, et al. Assessment of potential advantages of relevant ions for particle therapy: a model based study. Med Phys (2015) 42(2):1037-47. doi:10.1118/1.4905374

13. Hawkins RB. A microdosimetric-kinetic model for the effect of non-Poisson distribution of lethal lesions on the variation of RBE with LET. Radiat Res (2003) 160(1):61. doi:10.1667/RR3010

14. Inaniwa T, Suzuki M, Furukawa T, Kase Y, Kanematsu N, Shirai T, et al. Effects of dose-delivery time structure on biological effectiveness for therapeutic carbon-ion beams evaluated with microdosimetric kinetic model. Radiat Res (2013) 180(1):44-59. doi:10.1667/RR3178.1

15. Barendsen GW. Responses of cultured cells, tumours and normal tissues to radiations of different linear energy transfer. Curr Topics Radiat Res Q (1968) 4:293-356.

16. Furusawa Y, Fukutsu K, Aoki M, Itsukaichi H, Eguchi-Kasai K, Ohara H, et al. Inactivation of aerobic and hypoxic cells from three different cell lines by accelerated (3)He-, (12)C- and (20)Ne-ion beams. Radiat Res (2000) 154(5):485-96. doi:10.1667/0033-7587(2000)154[0485:IOAAHC]2.0.CO;2 Erratum in: Radiat Res. 2012 177(1):129-131.

17. Weyrather WK, Ritter S, Scholz M, Kraft G. RBE for carbon track-segment irradiation in cell lines of differing repair capacity. Int J Radiat Biol (1999) 75(11):1357-64. doi:10.1080/095530099139232
18. Belli M, Bettega D, Calzolari P, Cera F, Cherubini R, Dalla Vecchia M, et al. Inactivation of human normal and tumour cells irradiated with low energy protons. Int J Radiat Biol (2000) 76(6):831-9. doi:10.1080/09553000050028995

19. Sørensen BS, Overgaard J, Bassler N. In vitro RBE-LET dependence for multiple particle types. Acta Oncol (2011) 50(6):757-62. doi:10.3109/0284186X.2011. 582518

20. Hawkins RB. The relationship between the sensitivity of cells to high-energy photons and the RBE of particle radiation used in radiotherapy. Radiat Res (2009) 172(6):761-76. doi:10.1667/RR1655.1

21. Jones B, Underwood TC, Carabe-Fernandez A, Dale RG. Further analysis of fast neutron relative biological effects and implications for charged particle therapy. Br J Radiol (2011) 84:S11-8. doi:10.1259/bjr/67509851

22. Jones $B$. The apparent increase in the $\beta$-parameter of the linear quadratic model with increased linear energy transfer during particle irradiation. $\mathrm{Br} J$ Radiol (2010) 83(989):433-6. doi:10.1259/bjr/68792966

23. Friedrich T, Scholz U, Elsässer T, Durante M, Scholz M. Systematic analysis of $\mathrm{RBE}$ and related quantities using a database of cell survival experiments with ion beam irradiation. J Radiat Res (2013) 54(3):414-514. doi:10.1093/jrr/rrs114

24. Todd P. Heavy-ion irradiation of cultured human cells. Radiat Res (1967) 7:196-207. doi:10.2307/3583713

25. Goodhead DT. Inactivation and mutation of cultured mammalian cells by aluminium characteristic ultrasoft X-rays. III. Implications for theory of dual radiation action. Int J Radiat Biol Relat Stud Phys Chem Med (1977) 32:43-70. doi:10.1080/09553007714550751

26. Grassberger C, Trofimov A, Lomax A, Paganetti H. Variations in linear energy transfer within clinical proton therapy fields and the potential for biological treatment planning. Int J Radiat Oncol Biol Phys (2011) 80(5):1559-66. doi:10. 1016/j.ijrobp.2010.10.027

27. Paganetti H. Relative biological effectiveness (RBE) values for proton beam therapy. Variations as a function of biological endpoint, dose, and linear energy transfer. Phys Med Biol (2014) 59(22):R419-72. doi:10.1088/0031-9155/59/22/ R419

28. Jones B, Wilson P, Nagano A, Fenwick J, McKenna G. Dilemmas concerning dose distribution and the influence of relative biological effect (RBE) in proton beam therapy of medulloblastoma. Br J Radiol (2012) 85:912-8. doi:10.1259/ bjr/24498486

29. Holzscheiter MH, Bassler N, Dosanjh M, Sørensen BS, Overgaard J. A community call for a dedicated radiobiological research facility to support particle beam cancer therapy. Radiother Oncol (2012) 105(1):1-3. doi:10.1016/j.radonc. 2012.08.005

30. Dosanjh M, Jones B, Myers S. A possible biomedical facility at CERN. Br J Radiol (2013) 86(1025):20120660. doi:10.1259/bjr.20120660

31. Suit H, DeLaney T, Goldberg S, Paganetti H, Clasie B, Gerweck L, et al. Proton vs carbon ion beams in the definitive radiation treatment of cancer patients. Radiother Oncol (2010) 95:3-22. doi:10.1016/j.radonc.2010.01.015

Conflict of Interest Statement: The author declares that the research was conducted in the absence of any commercial or financial relationships that could be construed as a potential conflict of interest.

Copyright $\odot 2015$ Jones. This is an open-access article distributed under the terms of the Creative Commons Attribution License (CC BY). The use, distribution or reproduction in other forums is permitted, provided the original author(s) or licensor are credited and that the original publication in this journal is cited, in accordance with accepted academic practice. No use, distribution or reproduction is permitted which does not comply with these terms. 